\title{
Distribuição da hipovitaminose A no Brasil nas últimas quatro décadas: ingestão alimentar, sinais clínicos e dados bioquímicos
}

\section{Distribution of hipovitaminosis A in Brazil in the last four decades: dietary intake, clinical signs and biochemical data}

\author{
Rosana Regina Cardoso GERALDO ${ }^{1}$ \\ Sergio Alberto Rupp PAIVA² \\ Alexander Marcellus Carregosa da Silva PITAS ${ }^{3}$ \\ Irma GODOY2 \\ Álvaro Oscar CAMPANA²
}

\section{RE S U M O}

O presente estudo teve como objetivo o registro e a apresentação de trabalhos realizados no Brasil nos últimos 40 anos, relacionados com a investigação sobre a deficiência de vitamina A. Esta deficiência tem sido diagnosticada por um ou mais dos seguintes critérios: ingestão deficiente de alimentos fontes de vitamina $A$, exame clínico, níveis séricos de retinol abaixo dos aceitos como normais, concentração hepática de retinol, teste de adaptação ao escuro e corante de Rosa Bengala. A deficiência foi diagnosticada em grupos populacionais de vários Estados e capitais brasileiras em cidades grandes e pequenas e em zonas rurais. A maioria dos trabalhos foi desenvolvida em grupos populacionais de baixa renda. Quanto às conseqüências clínicas, relataram-se achados de sinais oculares leves, como cegueira noturna, manchas de Bitot e xerose conjuntival, encontrados principalmente na Região Nordeste. Alguns autores observaram, em menor número de casos, lesões graves, como lesões corneanas e ceratomalácia. Trabalhos da última década indicaram associação entre a hipovitaminose A e o aumento da morbidade e mortalidade, principalmente em crianças pré-escolares.

Termos de indexação: hipovitaminose A, xeroftalmia, retinol sérico, ingestão alimentar, cegueira noturna, retinol hepático, mortalidade e morbidade infantil.

\footnotetext{
1 Serviço Técnico de Nutrição e Dietética, Hospital das Clínicas, Faculdade de Medicina de Botucatu, Universidade Estadual Paulista.

2 Departamento de Clínica Médica, Faculdade de Medicina de Botucatu, Universidade Estadual Paulista. Rubião Junior, 16868-000, Botucatu, SP, Brasil. Correspondência para/Correspondence to: A.O. CAMPANA. E-mail: alvaro@fmb.unesp.br

3 Acadêmico, Curso de Nutrição, Instituto de Biociências do Campus de Botucatu, Universidade Estadual Paulista.
} 


\section{A B S T R A C T}

This work presents a review of investigations carried out in Brazil in the last 40 years, concerning the diagnosis of hypovitaminosis A. Vitamin A deficiency has been established by means of dietary intake, clinical signs, low serum and hepatic retinol concentrations, and by the rose bengal staining test and rapid dark-adaptation test. The vitamin deficiency has been found in many Brazilian, in many states capitals, in big and small cities and also in rural areas. Most of the research work has been undertaken with samples of populations of low socioeconomic level. As far as clinical signs are concerned, eye signs indicative of xerophtalmia were found mainly in the Northeastern Region. They included night blindness and conjunctival xerosis with or without Bitot's spots. More severe lesions such as irreversible changes or keratomalacia were rarely observed. Recent papers have shown the relationship between vitamin A deficiency and the increase in morbidity and mortality, mainly in pre-school children.

Index terms: hypovitaminosis A, xerophtalmia, serum retinol, hepatic retinol, dietary intake, night blindness, children morbidity and mortality.

\section{N T R O D U Ç Ã O}

A deficiência de vitamina $A$ é um dos problemas nutricionais mais freqüentes no mundo. A Organização Mundial da Saúde estimou que mais de 250 milhões de crianças em todo o mundo têm reservas diminuídas de vitamina $A^{1}$. Prevalência elevada desta carência é encontrada em crianças pré-escolares e em mulheres grávidas e lactantes ${ }^{2,3}$. A deficiência clínica da vitamina é definida pela presença de cegueira noturna, manchas de Bitot, xerose e/ou ulcerações corneanas e cicatrizes corneanas relacionadas à xeroftalmia ${ }^{4}$.

Há de se considerar, também, a deficiência subclínica de vitamina $A$, a qual, em crianças em idade pré-escolar, é definida pela prevalência de níveis de retinol sérico menores que 0,70 $\mu \mathrm{mol} / \mathrm{L}^{4}$. De acordo com a Organização Mundial da Saúde, o encontro de prevalência de níveis séricos de retinol $\leq 0,70 \mu \mathrm{mol} / \mathrm{L}$ em $2 \%$ a $<10 \%$ da população infantil de 6 a 71 meses de idade indica problema de saúde pública leve, de 10\% a <20\%, problema moderado e $\geq 20 \%$, grave $^{5}$.

A deficiência de vitamina A leva à ceratinização de epitélios. A ceratinização afeta não somente os olhos mas também o epitélio de revestimento dos tratos gastrintestinal, respiratório e do aparelho genitourinário. A ceratinização do epitélio gastrintestinal ou respiratório pode acarretar diminuição da resistência à colonização e à penetração de microorganismos ${ }^{6-7}$. A integridade do sistema imune pode também ser comprometida; um dos exemplos deste comprometimento seria a redução do transporte de imunoglobulinas secretoras através do epitélio alterado, respiratório ou gastrintestina| ${ }^{6-7}$. Estas alterações explicariam a associação freqüentemente descrita entre diarréia e infecções respiratórias e a deficiência de vitamina $A$.

As implicações da deficiência de vitamina A variam de acordo com o grupo de risco. Em crianças em idade pré-escolar, este distúrbio nutricional pode causar aumento do risco de mortalidade, morbidade e cegueira. Em mulheres grávidas e lactantes, a deficiência pode levar à cegueira noturna e parece ter implicações também na elevação da taxa de morbidade e mortalidade materna. As conseqüências para crianças em idade escolar e adolescentes têm sido menos estudadas e são provavelmente menos intensas ${ }^{8}$.

Não existem dados que permitam estabelecer a prevalência e a gravidade da deficiência de vitamina A para a população brasileira. Contudo, dispõe-se de apreciável número de estudos, realizados em várias regiões do Brasil, que fornecem indicações sobre a situação nutricional relacionada a esta vitamina. Revisões sobre esse tema foram publicadas por 
Dutra de Oliveira \& Marchini (1994) ${ }^{9}$ e Dutra de Oliveira et al. $(1996)^{10}$.

Em um número significativo dessas pesquisas, a hipovitaminose A foi reconhecida como um problema de saúde pública. Essa deficiência tem sido definida por meio de indicadores clínicos específicos, como os sinais oculares, e pelas alterações bioquímicas 5 . Do ponto de vista bioquímico, ela é considerada um problema de saúde pública quando $15 \%$ ou mais dos indivíduos apresentam concentrações de retinol no sangue menores que $20 \mu \mathrm{g} / \mathrm{dl}(0,70 \mu \mathrm{mol} / \mathrm{L})$ ou quando $5 \%$ ou mais da população tem concentrações menores que $10 \mu \mathrm{g} / \mathrm{dl}(0,35 \mu \mathrm{mol} / \mathrm{L})^{11}$.

O presente texto representa uma contribuição atualizada para o conhecimento da extensão da hipovitaminose A no Brasil. Este estudo teve como objetivo registrar os trabalhos originais, a partir da década de 60 , os quais correspondem a: 1) artigos originais publicados em periódicos científicos da área de ciências da saúde, de circulação nacional ou internacional, sobre alterações do estado nutricional relacionado com a vitamina A em grupos populacionais; 2) capítulos de livro e documentos publicados por instituições governamentais ou resultantes de parceria de instituições universitárias e governamentais. No caso dos artigos originais, deu-se ênfase às conclusões gerais alcançadas e procurou-se registrar algumas características dos grupos envolvidos, como idade, tamanho do grupo amostral e condições socioeconômicas.

\section{A L T E R A ÇÕ ES D O EST A D O NUTRICIONALRELACIONADO COM A VITAMINAA}

\section{A hipovitaminose A no Brasil nas décadas de 1960 a 1990}

\section{Década de 1960}

Pereira et al. (1966) ${ }^{12}$ estudaram 64 crianças de zero a 6 anos de idade (42 meninos e
22 meninas), com graus variados de desnutrição, em Florianópolis, SC. As crianças foram avaliadas por sinais e sintomas da hipovitaminose $A$, tendo-se encontrado 1 caso de cegueira noturna, 16 casos de xeroftalmia conjuntival e 45 casos de ceratomalácia.

Batista Filho \& Gomes (1969) ${ }^{13}$ publicaram os resultados de estudo realizado, em 1967, em duas comunidades da Zona da Mata do Nordeste do Brasil, o qual consistiu na dosagem de caroteno e vitamina A no soro, em 430 amostras, obtidas de indivíduos de várias faixas etárias (de 1 até 50 anos ou mais). Os valores médios da concentração sérica de vitamina $A$ se situavam acima de $30 \mu \mathrm{g} / \mathrm{dL}$, indicando ingestão satisfatória da vitamina ou de suas substâncias pré-formadoras. Contudo, a distribuição percentual dos níveis sangüíneos indicou a existência de baixas concentrações de vitamina $A(<20 \mu \mathrm{g} / \mathrm{dL})$ em $14,2 \%$ das crianças menores de 5 anos de idade. Foi registrado, também, que nenhuma criança menor de 4 anos ostentava carotenemia superior a $100 \mu \mathrm{g} / \mathrm{dL}$, e que, no mesmo grupo, $44,9 \%$ apresentavam níveis abaixo dos limites considerados como sugestivos de ingestão satisfatória de vegetais verdes e amarelos. Como conclusão, os autores assinalaram a maior vulnerabilidade dos grupos de mais baixa idade aos riscos da hipovitaminose $A$.

\section{Década de 1970}

Gomes et al. (1970) ${ }^{14}$, em 427 crianças em idade pré-escolar residentes em três cidades da "zona da mata", em Pernambuco, estudaram a associação de desnutrição protéico-calórica com os níveis séricos de retinol. Verificaram valores de retinol sérico baixos (10 a $19 \mu \mathrm{g} / \mathrm{dL}$ ) e deficientes $(<10 \mu \mathrm{g} / \mathrm{dL})$ em 10,2\% de crianças normais, em $14,9 \%$ de crianças com desnutrição de $1^{\circ} \mathrm{grau}$, em $23,6 \%$ daquelas com desnutrição de $2^{\circ}$ grau e em $53,8 \%$ daquelas com desnutrição de $3^{\circ} \mathrm{grau}$.

Varela et al. (1972) ${ }^{15}$, em pesquisa abrangendo 15 localidades do Nordeste brasileiro, com um total de 333 famílias, observaram que $4 \%$ das crianças pré-escolares apresentavam 
retinol sérico abaixo de $10 \mu \mathrm{g} / \mathrm{dL}$; na presença de desnutrição de $2^{\circ}$ e $3^{\circ} \mathrm{grau}$, esta porcentagem se elevava para $7 \%$. De maneira geral, 18,0\% das crianças pré-escolares mostravam níveis de retinol inferiores a $20 \mu \mathrm{g} / \mathrm{dL}$; quando havia desnutrição de $2^{\circ}$ e $3^{\circ} \mathrm{grau}$, a porcentagem subia a 33,0\%. Ainda de acordo com os autores, o consumo médio de vitamina A era baixo e os sintomas atribuídos à hipovitaminose A estavam presentes em crianças de 1 a 4 anos (xerose conjuntival: 0,3\% e manchas de Bitot: 0,2\%) e em jovens de 10 a 19 anos de idade (xerose conjuntival: 1,2\%). Entre as crianças desnutridas de 1 a 4 anos de idade, a xerose conjuntival acometia 1,0\% delas. Cegueira noturna foi encontrada em 3 casos; entretanto, foi referido que esta freqüência poderia estar subestimada, em virtude da dificuldade de coletar os dados em menores de 5 anos de idade.

Um estudo incluindo inquérito alimentar, pesquisa de sinais clínicos de hipovitaminose $\mathrm{A}$ e dosagem dos níveis séricos de vitamina $A$ e caroteno foi realizado em três localidades do Estado de São Paulo - Iguape, Icapara e Pontal do Ribeira ${ }^{16}$. A dosagem de retinol no sangue foi feita em 343 indivíduos de ambos os sexos. Verificaram-se: 1) valores deficientes de retinol sérico ( $<10 \mu \mathrm{g} / \mathrm{dL}$ ) em 8,2\% da amostra; 2 ) valores

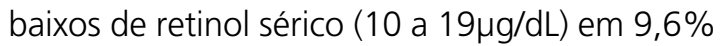
da amostra. Os autores concluíram que, em Iguape, Icapara e Pontal de Ribeira, a deficiência de vitamina A representava um problema de saúde pública.

Batista Filho et al. (1973) $)^{17}$ avaliaram o estado nutricional relacionado à vitamina $\mathrm{A}$ em gestantes de duas localidades urbanas da zona da mata de Pernambuco (Água Preta e Gameleira). Dentre os 159 casos estudados, verificaram-se níveis altos de retinol em 49,1\% e níveis aceitáveis (20 a 49 $\mathrm{g} / \mathrm{dL}$ ) em 48,4\% deles;

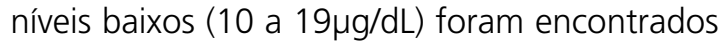
em apenas quatro casos (2,5\%). Desta maneira, os autores concluíram que, no grupo estudado, "a hipovitaminose A não deveria ser considerada um problema nutricional de imediata prioridade".
Segundo os estudos de Bon \& Miguel $(1974)^{18}$ e Mazzili (1975) ${ }^{19}$, realizados na mesma área geográfica, o consumo médio diário de vitamina $A$ mostrou-se inadequado em amostra constituída de 28 famílias (197 pessoas) do município de Ribeira e em 105 famílias de Icapara (consumo médio diário com adequação em 10\% dos casos) e 56 famílias de Pontal do Ribeira (consumo médio diário com adequação de 15\%).

Investigação que incluiu a avaliação do consumo alimentar por pesagem de alimentos, associada a método recordatório, conduzida em amostra de 194 famílias de lguape, SP, identificou o consumo médio de vitamina A de $549 \mu \mathrm{g}$ e uma adequação de $41 \%$. Em linhas gerais, o consumo médio per capita de vitamina A foi baixo, sendo deficiente para a maioria das famílias analisadas ${ }^{20}$.

Níveis séricos de retinol e caroteno abaixo dos valores aceitáveis foram também encontrados em migrantes atendidos pela Central de Triagem e Encaminhamento da Capital do Estado de São Paulo ${ }^{21,22}$. Níveis deficientes $(<10 \mu \mathrm{g} / \mathrm{dL})$ de vitamina A foram observados em $13,9 \%$ das crianças de 2 a 7 anos e em 8,2\% de adolescentes e adultos do sexo masculino e em $9,3 \%$ do sexo feminino; níveis baixos (10 a 19,9 $\mathrm{g} / \mathrm{dL}$ ) foram encontrados em $37,5 \%$ das crianças de 2 a 7 anos, em $14,1 \%$ de adolescentes e adultos do sexo masculino e em $23,4 \%$ do sexo feminino. Níveis deficientes $(<20 \mu \mathrm{g} / \mathrm{dL})$ de caroteno foram detectados em 10,3\% das crianças e em 2,7\% dos adolescentes e adultos do sexo masculino e em $2,4 \%$ do sexo feminino; quanto aos níveis baixos (20 a 39,9 $\mu \mathrm{g} / \mathrm{dL}$ ), foram observados em $47,4 \%$ das crianças e em $24,1 \%$ de adolescentes e adultos do sexo masculino e $15,6 \%$ do sexo feminino. De acordo com a análise do consumo dos alimentos, avaliado pelo método recordatório, a ingestão de alimentos fontes de vitamina $A$ e carotenóides era insatisfatória ${ }^{23}$. Quanto aos sinais clínicos das 109 crianças de 2 a 7 anos de idade examinadas, 80,7\% apresentavam manifestações atribuíveis à hipovitaminose $A^{22}$. Dentre os sinais oculares, apenas a xerose conjuntival mereceu destaque, estando presente em 13,8\% dos 
examinados. Registrou-se, ainda, um caso de xerose corneana. Assim, os dados indicaram que a xeroftalmia não era problema de saúde pública no grupo estudado, embora os resultados do exame bioquímico mostrassem que a hipovitaminose $\mathrm{A}$ o era ${ }^{22}$.

Esses resultados motivaram os autores a realizar um levantamento sobre a prevalência da xeroftalmia em Estados do Brasil, utilizando, como instrumento, questionários que deveriam ser respondidos por oftalmologistas. A devolução dos questionários foi pequena, mas registrou-se baixa freqüência de manifestações oculares. Desta maneira, estes achados ${ }^{24}$ estão em frontal desacordo com os dados relatados por Simmons \& Melo $(1975)^{25}$ e Simmons $(1976)^{26}$ sobre lesões oculares na hipovitaminose $A$. Estes últimos autores publicaram dados concernentes à xeroftalmia e à cegueira noturna, observadas em nove Estados do Nordeste brasileiro. O estudo consistiu, fundamentalmente, na análise dos registros de pacientes ambulatoriais e internados em hospitais de cidades daquela Região do Brasil. Os autores concluíram que a xeroftalmia constituía um problema de saúde pública no Nordeste brasileiro e era uma causa de cegueira em certas áreas. Simmons $(1976)^{26}$, assinalou haver limitações ao trabalho em pauta, como a não-especificidade dos sinais clínicos leves de hipovitaminose $A$, o fato de os dados terem sido obtidos por meio de registros hospitalares ou por entrevistas com médicos da região e a diferença do nível de atendimento médico entre os vários locais estudados.

Em estudo incluindo exame clínico, antropometria e inquérito de consumo de alimentos, Giugliano et al. (1978)27 observaram, em 69 escolares de 7 a 11 anos, habitantes de Manaus, hiperqueratose folicular e xerose $(29,0 \%$ dos casos), espessamento conjuntival $(81,1 \%$ dos casos) e mancha de Bitot (em um caso). Estes achados, segundo os autores, indicariam a existência de provável deficiência de vitamina $A$.

Logo a seguir, Shrimpton \& Giugliano $(1979)^{28}$ publicaram os resultados do consumo de alimentos provenientes da primeira Pesquisa sobre Orçamentos Familiares, envolvendo 1200 famílias de Manaus, realizada em 1973 e 1974. Estes autores verificaram que a dieta era deficiente em vitamina $\mathrm{A}$.

Em Belo Horizonte, MG, Araujo et al. $(1978)^{29}$ realizaram estudo para verificar o efeito da ingestão diária de açúcar fortificado com vitamina A em crianças pré-escolares provenientes de classe socioeconômica baixa, cuja dieta era pobre em vitamina A. Para isso, constituíram dois grupos de crianças de 8 a 89 meses de idade, atendidas em orfanato. Nesses grupos, antes da suplementação vitamínica, foram observados níveis de retinol plasmático deficientes $(<10 \mu \mathrm{g} / \mathrm{dL})$ em $54,0 \%$ e $56,0 \%$ e níveis baixos (de $10 \mathrm{a}<20 \mu \mathrm{g} / \mathrm{dL}$ ) em $44,2 \%$ e $41,4 \%$ das crianças. Os autores relataram não ter encontrado nenhum sintoma ou sinal clínico de hipovitaminose $A$.

Em 141 pré-escolares de baixo nível socioeconômico de Ribeirão Preto, a disponibilidade da vitamina A na alimentação foi avaliada por Carvalho \& Dutra de Oliveira $(1979)^{30}$, por meio de levantamento dos alimentos recebidos nos parques infantis, com verificação das quantidades utilizadas e, também, através de inquérito alimentar qualitativo, realizado com as mães. $O$ consumo de vitamina A era aparentemente adequado. O exame clínico de 38 dessas crianças não revelou nenhuma manifestação atribuída à deficiência da vitamina. Não foi realizada a dosagem de vitamina $A$ no sangue, mas foi medido o nível de caroteno plasmático em oito crianças; observou-se baixa concentração de caroteno $(<20 \mu \mathrm{g} / \mathrm{dL})$ em apenas uma delas. Entretanto, como a maior parte da vitamina A ingerida foi proveniente de alimentos de origem vegetal, os autores levantaram a possibilidade de o grupo estudado constituir alvo de hipovitaminose $\mathrm{A}$.

\section{Década de 1980}

Em Fortaleza, a análise do consumo alimentar de 366 famílias, migrantes, de baixo 
nível econômico, indicou que mais de $90 \%$ das famílias não consumiam o requerimento mínimo diário de vitamina $\mathrm{A}^{31}$.

Em estudo envolvendo 100 famílias de bóias-frias da região de Ribeirão Preto, Desai et al. (1980)32 observaram que aproximadamente $25 \%$ dos integrantes da unidade familiar tinham baixos níveis plasmáticos de vitamina A (10 a $19 \mu \mathrm{g} / \mathrm{dL}$ ); com respeito aos níveis séricos de caroteno plasmáticos, aproximadamente 10\% foram deficientes e 20\%, baixos. Estes dados podem refletir a dieta consumida por este grupo populacional, baseada fundamentalmente em arroz e feijão, com uso pouco freqüente de produtos de origem animal e baixa ingestão de vegetais e frutas verdes e amarelas.

Em 27 crianças de um orfanato de São Paulo, com idade variando de 1 a 13 anos, verificaram-se níveis séricos de vitamina $A$ considerados baixos e deficientes em 59,1\% e $4,5 \%$ delas respectivamente; quanto ao caroteno sérico, níveis baixos foram encontrados em 22,7\% dos $\operatorname{casos}^{33}$.

No período de 1969 a 1973, foram realizados inquéritos nutricionais de consumo alimentar e dosagens bioquímicas de vitamina $A$ e caroteno em 1100 famílias de onze comunidades do Estado de São Paulo ${ }^{34}$. Verificou-se haver grande variação no consumo de vitamina $\mathrm{A}$, porém mais de $80,0 \%$ das famílias apresentavam níveis de adequação de consumo abaixo de 60,0\%. 0 exame clínico, realizado em 1660 indivíduos das onze comunidades, mostrou baixos coeficientes de prevalência de lesões oculares, principalmente mais graves. A porcentagem de manifestações oculares foi de $16,3 \%$. Nos 271 casos com lesões oculares, identificaram-se: xerose conjuntival $(15,5 \%)$, mancha de Bitot $(0,5 \%)$ e xerose corneal com xerose conjuntival $(0,3 \%)$. Em relação à dosagem bioquímica, empreendida em $75,0 \%$ dos indivíduos examinados clinicamente, observou-se que 5,4\% apresentavam níveis plasmáticos deficientes e 12,9\%, níveis baixos de vitamina A; em relação ao caroteno plasmático, foram encontrados níveis deficientes em $14,3 \%$ e baixos em 20,7\%. Assim, 5,4\% apresentavam níveis plasmáticos iguais ou inferiores a $10 \mu \mathrm{g} / \mathrm{dL}$ e $18,3 \%$, níveis iguais ou inferiores a $20 \mu \mathrm{g} / \mathrm{dL}$; isto levou os autores a admitirem que a deficiência de vitamina A era um problema de saúde pública na área estudada. Além disso, os pré-escolares apresentavam freqüência mais elevada em relação a outras faixas etárias de resultados tanto abaixo de $10 \mu \mathrm{g} / \mathrm{dL}$, como abaixo de $20 \mu \mathrm{g} / \mathrm{dL}$. Note-se que, mesmo na faixa de 7 a 10 anos, observaram-se níveis de vitamina A plasmática baixos em $23,1 \%$ e deficientes em $11,9 \%$ das crianças examinadas. Quanto ao caroteno plasmático, foram evidenciados níveis deficientes principalmente em crianças de 2 a 6 anos de idade, imediatamente seguidas por aquelas de 7 a 10 anos.

Em indivíduos de 50 anos ou mais, observaram-se níveis plasmáticos de vitamina $\mathrm{A}$ considerados deficientes em 3,8\% e baixos em 5,8\% dos 158 casos analisados. Desta maneira, esses resultados não caracterizaram um problema de saúde pública nesse grupo populacional. Ainda nesse grupo, com relação ao caroteno plasmático, foram encontrados $24,1 \%$ de níveis deficientes e $24,7 \%$, baixos. Estes resultados sugeriram que a ingestão recente de alimentos ricos em carotenóides, nessa amostra, era baixa ${ }^{35}$.

Em 20 crianças, em idade pré-escolar, provenientes de baixa classe socioeconômica e residentes em creche mantida pela Fundação Estadual do Bem-Estar do Menor (FEBEM) de cidade satélite de Belo Horizonte, as quais se alimentavam com dieta balanceada contendo $1300 \mathrm{UI}$ de vitamina A por dia, Borges et al. (1981) ${ }^{36}$ observaram valor médio de retinol sérico baixo $(10,5 \mu \mathrm{g} / \mathrm{dL})$. As crianças não apresentavam nenhum sinal clínico de deficiência de vitamina $A$.

Horner et al. (1981) 37 publicaram trabalho relacionado com inquérito realizado em população pobre da cidade de llhéus, na Bahia. A metodologia empregada consistiu em entrevista para verificação do consumo de todos os alimentos ingeridos em período de 24 horas por toda a família. A análise 
limitou-se a 31 famílias. Foi verificado que a dieta consumida era pouco variada e deficiente em vitamina A.

Em estudo conduzido nas margens do rio Solimões (Amazonas), o exame clínico de 140 crianças de zero a 5 anos de idade sugeriu a existência de deficiência de vitamina A em cerca de $20 \%$ delas; a hiperqueratose folicular e o espessamento conjuntival foram os sinais clínicos encontrados, sugestivos da deficiência ${ }^{38}$.

Outro estudo, desenvolvido com 153 pré-escolares de um bairro pobre de Manaus, mostrou um consumo alimentar monótono, mas com ingestão adequada de vitamina $A^{39}$.

Uma análise do consumo alimentar e da adequação da ingestão de nutrientes segundo o nível de renda familiar, envolvendo 1200 famílias, foi realizada em Manaus (Amazonas). Verificou-se que $40 \%$ a $50 \%$ das famílias de renda mais alta (mais de 5,2 salários mínimos) e 60\% a $80 \%$ das famílias cujo rendimento era baixo ou médio (até 5,2 salários mínimos) não conseguiam ingerir cotas seguras de vitamina $A(\geq 70 \%$ da ingestão dietética recomendada, de acordo com a World Health Organization de 1967) ${ }^{40,41}$.

Ainda no Estado do Amazonas, foram estudados 382 operários, de ambos os sexos, com idade entre 15 e 69 anos, em quatro fábricas de Manaus ${ }^{42}$. Em duas fábricas os níveis de retinol sérico eram inferiores a $10 \mu \mathrm{g} / \mathrm{dL}$ e em três fábricas mais de $15 \%$ dos operários tinham níveis de retinol abaixo de $20 \mu \mathrm{g} / \mathrm{dL}$. Os homens apresentavam níveis séricos deficientes $(<20 \mu \mathrm{g} / \mathrm{dL}$ ) e baixos (de 20 a $29 \mu \mathrm{g} / \mathrm{dL}$ ) de caroteno, em todas as faixas etárias; as mulheres, ao contrário, apresentavam níveis normais. Este quadro indicou que a deficiência de vitamina A constituía um problema de saúde pública importante para essa população.

No início da década de 80, foi realizado um estudo epidemiológico para levantamento da prevalência de sinais oculares da hipovitaminose A em 13404 crianças, de zero a 12 anos de idade, provenientes de oito municípios representativos das três regiões ecológicas do Estado da Paraíba.
Dentre os sinais clínicos foram observados: cegueira noturna, xerose conjuntival com mancha de Bitot e, com freqüência menor, xerose da córnea e cicatriz corneana. Estes sinais foram detectados quase majoritariamente nas crianças da região do semi-árido. Esses resultados indicaram que a deficiência de vitamina A era um problema de saúde pública na zona semi-árida da Paraíba ${ }^{43,44}$. Posteriormente, foi publicada a observação de cinco casos graves de xeroftalmia e ceratomalácia em crianças de 3 a 28 meses de idade, internadas em Hospital Universitário, na Paraíba. Registrouse, entre as crianças afetadas, casos de cegueira irreversível ${ }^{45}$

Em pesquisa realizada em Recife, envolvendo crianças menores de 7 anos (49 meninos e 44 meninas), provenientes de famílias de baixa renda ${ }^{46}$, encontraram os níveis séricos médios de retinol situados dentro da faixa de normalidade. Entretanto, havia dispersão dos valores de retinol sérico, cuja amplitude variou de 7,6 a 73,9 $9 \mu \mathrm{g} / \mathrm{dL}$, implicando na existência de crianças com níveis séricos indicativos de deficiência de vitamina $A$ Por outro lado, a utilização do teste Relative Dose Response (RDR) trouxe evidências de que valores de 20 a $30 \mu \mathrm{g} / \mathrm{dL}$ de retinol sérico poderiam coexistir com deficiência marginal de vitamina A, principalmente quando a história dietética sugeria risco de deficiência.

Em Ribeirão Preto, Desai et al. (1984)47, analisando o consumo alimentar de 121 adolescentes, verificaram $50 \%$ de adequação na ingestão de vitamina A entre os jovens pertencentes a famílias de baixa renda, e de $131 \%$ no caso de famílias de melhor condição socioeconômica.

Em Cotia, SP, Roncada et al. (1984) ${ }^{48}$ observaram níveis séricos baixos e deficientes de vitamina A, respectivamente em $26,6 \%$ e em $3,6 \%$, entre 362 crianças de 3 a 6 anos de idade. Levando-se em conta critérios internacionalmente aceitos, a hipovitaminose $A$, na localidade estudada, era problema de saúde pública (30,2\% dos valores de retinol sérico abaixo de $20 \mu \mathrm{g} / \mathrm{dL}$ ). Adicionalmente, $11,6 \%$ das crianças tinham níveis 
de caroteno abaixo dos aceitos como normais $(<40 \mu \mathrm{g} / \mathrm{dL})$.

Em trabalho realizado com crianças em idade escolar e pré-escolar, provenientes de áreas pobres do Estado de Minas Gerais (Turmalina, Minas Novas e Capelinha, no Vale do Jequitinhonha), Araujo et al. (1986) ${ }^{49}$ verificaram que a hipovitaminose A era um problema de saúde pública nessas populações. Os autores observaram valores deficientes de retinol sérico $(<10 \mu \mathrm{g} / \mathrm{dL})$ em $8,9 \%$ de 67 crianças pré-escolares e em 4,4\% de 90 crianças em idade escolar, na zona urbana das três cidades mencionadas. Quanto à zona rural, foram avaliadas somente as crianças de Turmalina, tendo-se encontrado valores deficientes apenas em crianças pré-escolares $(5,8 \%$ de total de 52 crianças). Em Belo Horizonte, Araujo et al. (1987) (50 $^{5}$ observaram, também, prevalência elevada de valores deficientes $(<10 \mu \mathrm{g} / \mathrm{dL}$ ) e baixos (de $10 \mathrm{a}$ $20 \mu \mathrm{g} / \mathrm{dL}$ ) de retinol sérico em $21,5 \%$ de 130 crianças pré-escolares e $21,7 \%$ de 120 crianças em idade escolar, provenientes de estratos socioeconômicos baixos.

Em 1980, foi desenvolvido um estudo com a finalidade de analisar a situação alimentar e nutricional da população de quatro municípios de Pernambuco, classificada em quatro categorias, de acordo com o acesso e o tamanho da propriedade. Assim, foram categorizadas em famílias sem terra (ST), famílias de pequenos proprietários, que possuíam de zero a 10 hectares (PP), de médios proprietários, cuja posse de terra era de 10 a 50 hectares (MP), e de grandes proprietários, os quais possuíam mais de 50 hectares (GP). Para quantificar o consumo alimentar, foi utilizado o método recordatório e a pesagem direta dos alimentos (cobertura de um dia) de 689 famílias $^{51}$. A adequação média da ingestão de vitamina A no grupo sem terra foi de $69,1 \%$ e nos outros grupos foi superior a 100,0\%. Além disso, analisando os grupos em conjunto, porcentagens diferentes de famílias não atingiam o limite de $50,0 \%$ da recomendação da ingestão de vitamina $A$; estas porcentagens eram maiores nos grupos mais pobres e distribuíram-se assim: 81,6\% no grupo ST, 75,6\% no grupo PP, 61,8\% no grupo MP e 30,8\% no grupo GP. Neste trabalho, analisou-se, também, a distribuição da hipovitaminose A em crianças com menos de 6 anos de idade, de acordo com o acesso à terra e o tamanho da propriedade de suas famílias. Participaram do estudo 1257 crianças $^{52}$. Os resultados indicaram a existência de valores baixos de retinol em 17,2\% delas e valores deficientes em $6,8 \%$ das crianças, totalizando a prevalência de hipovitaminose A de 24,0\%. Não foi observada associação significante entre os níveis séricos de vitamina $\mathrm{A}$ e as diferentes categorias de posse de terra.

No Estado da Paraíba, inquérito alimentar do tipo recordatório de 24 horas foi aplicado a uma população de baixa renda, habitante da periferia de João Pessoa e da cidade de Bananeiras ${ }^{53}$. Estabeleceram-se, então, os valores estimados de vários nutrientes, para 151 crianças, de acordo com as seguintes faixas etárias: 1 a 3, 4 a 6 e 7 a 9 anos. Concluiu-se que a dieta ingerida era quantitativamente e qualitativamente deficiente em vários nutrientes, particularmente em vitamina $A$, cuja ingestão média era inferior a $50 \%$ das necessidades fisiológicas.

Em crianças em idade pré-escolar, pertencentes a famílias de baixo nível socioeconômico e residentes em bairros periféricos de Ribeirão Preto, SP, verificaram-se níveis deficientes de retinol plasmático em $1,8 \%$ e baixos em $47,2 \%$ delas. Os testes de adaptação ao escuro e Rosa Bengala, porém, não indicaram a existência de xerose conjuntival e cegueira noturna. Os autores não registraram nenhum caso de xeroftalmia nas crianças estudadas $^{54,55}$. No entanto, chamaram a atenção para o fato de que, embora não houvesse casos graves de hipovitaminose A na população estudada, a deficiência de vitamina A constituia um problema de saúde pública nessa região, pois cerca de metade das crianças examinadas tinha níveis baixos $(<20 \mu \mathrm{g} / \mathrm{dL})$ de vitamina A plasmática.

Mariath et al. (1989) ${ }^{56}$ relataram observações realizadas em 6291 crianças de até 12 anos de idade, procedentes de três localidades 
da região semi-árida do Rio Grande do Norte. Verificaram a ocorrência de manchas de Bitot e xerose conjuntival em $0,3 \%$ a $1,4 \%$ das crianças examinadas; entretanto, cegueira noturna foi encontrada em apenas três casos. Em 44 crianças, foi realizado o teste da resposta relativa à dose (RDR); o teste revelou-se positivo em 16 casos.

\section{Década de 1990}

No estudo de um pequeno grupo populacional de gestantes $(n=25)$, atendidas em Centro de Saúde da periferia de Campinas, verificou-se deficiente ingestão de vitamina $\mathrm{A}$ em $50 \%$ delas $^{57}$.

Em pesquisa realizada em área rural de município da Bahia, foram determinados os valores de retinol sérico ${ }^{58}$ e foi analisada a associação de deficiência de vitamina $A$ com a desnutrição energético-protéica ${ }^{59}$ em 161 crianças de 6 a 72 meses de idade. A prevalência de desnutrição atingiu índices de 1,9\%, 15,5\% e 32,9\% conforme o indicador utilizado para o diagnóstico da desnutrição (peso/altura, peso/idade e altura/idade, respectivamente); foram observados níveis séricos de retinol baixos em $40,4 \%$ e deficientes em $4,3 \%$ dos casos; assim, 44,7\% das crianças apresentavam níveis inadequados de vitamina $\mathrm{A}$. Desta maneira, este achado caracterizou a deficiência como um problema de saúde pública. Os resultados também indicaram que a desnutrição energético-protéica não se mostrou associada à deficiência de vitamina A. Entretanto, as crianças com déficit de altura/idade apresentavam percentuais mais elevados de níveis séricos de vitamina A considerados inadequados.

Santos et al. (1996) ${ }^{60}$ publicaram os resultados de estudo envolvendo amostra de 754 pré-escolares de áreas urbanas de sete municípios do semi-árido do Estado da Bahia. Não foram observados sintomas e/ou sinais oculares de hipovitaminose $A$. Os autores observaram que apenas $8 \%$ das crianças consumiam quantidades adequadas de retinol ou de seus precursores. Em todos os municípios, a prevalência de retinol sérico deficiente foi superior a 5,0\%; considerando o número total de amostras de sangue colhido ( $n=563)$, a prevalência de níveis séricos deficientes de retinol $(<0,35 \mu \mathrm{mol} / \mathrm{L})$ foi de $15,3 \%$. A distribuição do retinol sérico não se mostrou associada com o sexo, mas apresentou correlação com a idade das crianças, havendo diminuição dos níveis deficientes e baixos à medida que aumentava a idade das crianças. Também não se encontrou associação entre a renda familiar per capita e a prevalência dos níveis deficientes de retinol. Os dados em conjunto indicaram que a carência de vitamina A, na população estudada, devia ser considerada como um problema grave de saúde pública.

O consumo da vitamina A foi avaliado por meio de entrevista elaborada de acordo com a metodologia que aborda o histórico alimentar individual, em população de adultos, residentes em Cotia, a partir de subamostra de 548 indivíduos com idade entre 20 e 88 anos $^{61}$. Verificou-se que a dieta habitual dessa população apresentava-se inadequada com relação à vitamina $\mathrm{A}$.

A hipovitaminose A foi investigada em recém-nascidos, em duas maternidades públicas, no Rio de Janeiro, tal como publicado por Ramalho et al. (1998)62. O retinol sérico foi determinado em sangue do cordão umbilical de 253 recém-nascidos, cujas mães eram procedentes de áreas consideradas de baixa condição socioeconômica. A maioria deles $(55,7 \%)$ apresentou valores séricos de retinol inferiores a $1,05 \mu \mathrm{mol} / \mathrm{L}$ (ou $30 \mu \mathrm{g} / \mathrm{dL}$ ), ponto de corte utilizado, neste estudo, para indicar hipovitaminose A. Não foi observada qualquer associação entre o estado nutricional avaliado antropometricamente e níveis de retinol sérico dos recém-nascidos.

A ingestão de alimentos fontes de vitamina A foi avaliada em 74 nutrizes de 14 a 39 anos de idade, distribuídas em três grupos: dois grupos pertencentes a estratos de baixa condição socioeconômica (adolescentes e adultas) e o terceiro constituído por mulheres adultas de alta condição socioeconômica. A ingestão foi avaliada pelo método recordatório de 24 horas. Houve 
diferença significativa da ingestão de vitamina $A$ entre os três grupos, sendo obtidos valores menores nos grupos de baixa condição socioeconômica ( $281 \mu \mathrm{gER}$ e $412 \mu \mathrm{gER}$ ) e maior no grupo de alta condição socioeconômica $(770 \mu \mathrm{gER})^{63}$.

Um estudo de caso-controle desenvolvido em quatro hospitais de Campinas, no Estado de São Paulo, procurou avaliar a relação existente entre os níveis de vitamina $A$ do sangue materno e de sangue do cordão umbilical de recém-nascidos, nos casos em que o peso do recém-nascido era adequado e naqueles em que o peso era menor ${ }^{64}$. Fizeram parte da pesquisa 356 pares de mães e recém-nascidos com retardo de crescimento e 356 pares de mães e recém-nascidos com peso adequado. Foi notada marcante diferença entre os valores de retinol do sangue do cordão umbilical de crianças com baixo peso (33,1\% delas apresentando valores $\leq 0,70 \mu \mathrm{mol} / \mathrm{L})$ e de crianças com peso adequado (14,9\% delas apresentando valores $\leq 0,70 \mu \mathrm{mol} / \mathrm{L}$ ). Entretanto, 98,7\% das mães apresentavam níveis sangüíneos dentro da normalidade. De acordo com os resultados obtidos, a deficiência de vitamina A não constituía um problema de saúde na população materna estudada e havia uma associação entre a deficiência de vitamina A no recém-nascido e o baixo peso ao nascer ${ }^{65}$. Nesta mesma população, Rondó et al. (1999)66 empregaram um questionário de freqüência alimentar, incluindo 55 alimentos da dieta típica de famílias de baixo nível socioeconômico do sudeste brasileiro. Usando critério que permitiu classificar o consumo de vitamina A em três categorias, de acordo com o risco de desenvolver deficiência de vitamina $A$ (ingestão de vitamina $A \geq 481 \mu \mathrm{gER} /$ dia $=$ risco baixo; ingestão de 480 a $221 \mu \mathrm{gER} / \mathrm{dia}=$ risco moderado; ingestão $\leq 220 \mu \mathrm{gER} / \mathrm{dia}=$ risco alto), os autores puderam afirmar que a maioria das mulheres envolvidas no estudo não tinham risco alto de desenvolver deficiência de vitamina $A$.

Níveis de retinol sérico iguais ou abaixo de $0,70 \mu \mathrm{mol} / \mathrm{L}$ foram observados em $21,4 \%$ de 103 crianças sadias, de 6 a 24 meses de idade, acompanhadas em ambulatório de Puericultura em Ribeirão Preto ${ }^{67}$. As crianças pertenciam à classe média, não apresentavam sinais de xeroftalmia, infecção aguda e/ou diarréia. A porcentagem de valores séricos deficientes está acima do índice de $20 \%$, estabelecido como indicador de problema grave de saúde pública. Este trabalho não mostrou associação entre renda familiar e deficiência de vitamina A, diagnosticada por diminuição da concentração sérica de retinol. Os autores, entretanto, chamaram a atenção para a ausência de uma variação ampla da renda familiar.

\section{Níveis de Retinol Hepático e Hipovitaminose A}

Um estudo realizado em Salvador, Bahia, no fim da década de 70, trouxe evidências de esgotamento das reservas hepáticas de retinol em $30 \%$ das crianças de 3 a 24 meses de idade que morreram por diversas causas ${ }^{68}$. Posteriormente, em Recife, Flores \& Araujo (1984) ${ }^{69}$, em investigação incluindo a análise de 517 crianças de 0 a 14 anos de idade, falecidas devido a várias causas, verificaram que a idade e o estado nutricional eram os principais determinantes de baixas reservas hepáticas de vitamina $A$. De acordo com estes autores, as crianças com menos de um ano de idade apresentavam prevalência mais elevada de valores deficientes de retinol hepático em relação aos valores observados nas outras faixas etárias. Adicionalmente, após o primeiro ano de vida, a desnutrição energético-protéica associava-se à maior prevalência de comprometimento da reserva hepática de retinol.

Os autores extrapolaram seus dados para a população viva com menos de 5 anos de idade. Tendo usado o critério de que reservas hepáticas inferiores a 5 e $20 \mu \mathrm{g}$ de retinol por grama de fígado representam, respectivamente, situações de alto risco e inadequação do estado nutricional relativo à vitamina $\mathrm{A}$, concluíram que aproximadamente $3 \%$ e $17 \%$ das crianças ( 0 a 4 anos de idade) de Recife poderiam ser incluídas em grupos 
de alto risco e de inadequação, respectivamente, no tocante à hipovitaminose $A^{69}$.

Em 1981, foi realizado estudo em Brasília, no qual foi analisada a concentração de vitamina A no fígado de 57 fetos e crianças que morreram devido a várias causas ${ }^{70}$, procedentes de famílias indigentes $(n=22)$ e não indigentes $(n=35)$. Os autores observaram que $12 \%$ das crianças tinham concentrações inferiores a $20 \mu \mathrm{g} / \mathrm{g}$ de fígado, denotando reserva inadequada da vitamina embora nenhuma criança tivesse apresentado valores de concentração hepática de retinol inferiores a $5 \mu \mathrm{g} / \mathrm{g}$. Os resultados demonstraram valores médios e medianas de concentração de vitamina A das amostras de fígado de crianças provenientes de famílias indigentes menores do que os valores correspondentes em crianças de famílias não indigentes. Entretanto, esses números encontravam-se acima dos valores usados para indicar diminuição da reserva hepática de vitamina $A^{69}$.

\section{Deficiência de Vitamina A, Infecções e Infestação Parasitária}

Estudos investigativos têm mostrado a inter-relação entre infecção e infestação por parasitas e hipovitaminose A. Araujo et al. $(1978)^{71}$, por exemplo, referiram a importância de se considerar a existência de parasitose intestinal na hipovitaminose A. Em 1988, foi publicado trabalho que estuda a inter-relação entre a infestação pelo Schistosoma mansoni e o nível sérico de retinol de população de município próximo de Belo Horizonte $(\mathrm{MG})^{72}$. Foram encontrados níveis de retinol sérico abaixo dos aceitáveis $(20 \mu \mathrm{g} / \mathrm{dL})$ em $60,4 \%$ dos casos estudados (106 indivíduos). Na investigação, porém, não foram observados sinais clínicos compatíveis com hipovitaminose A. Além disso, a distribuição entre níveis de retinol sérico aceitáveis e não aceitáveis em dois grupos estudados (grupo infectado por S. Mansoni e grupo controle), analisada pelo teste do qui-quadrado, não mostrou diferença estatisticamente significante entre os grupos. Também não se observou correlação significante entre a carga parasitária e o nível de retinol sangüíneo ${ }^{72}$.

Entretanto, em uma amostra de 240 crianças de 3 a 7 anos, provenientes de distrito pobre de Manaus, que apresentavam infestação intestinal por Ascaris lumbricoides e/ou Giardia lamblia, verificaram-se níveis médios de retinol e caroteno séricos abaixo dos valores normais, respectivamente, em $36 \%$ e $57 \%$ dos $\operatorname{casos}^{73}$.

O efeito da infecção sobre o estado nutricional relacionado à vitamina $A$ foi relatado em estudo de Campos et al. (1987)74, no qual a alta prevalência da positividade do teste RDR, após episódio de varíola em crianças, sugeriu depleção da reserva hepática dessa vitamina.

Os níveis plasmáticos de vitamina $A$, carotenóides e proteína ligadora do retinol foram estudados em 311 crianças de 7 meses a 11 anos de idade, em Pronto-Socorro Infantil e em enfermaria pediátrica de hospital municipal de São Paulo. Com base no diagnóstico clínico, constituíram-se quatro grupos de estudo: um primeiro grupo, representado por crianças com infecção das vias respiratórias superiores; o segundo, com diarréia aguda; o terceiro, com pneumonia ou broncopneumonia; e o quarto, correspondendo ao grupo testemunha ${ }^{7}$. Os níveis plasmáticos de vitamina A foram mais baixos que os do grupo controle nos grupos com pneumonia e diarréia e os níveis de carotenóides foram mais baixos nos três grupos com afecção clínica. Níveis diminuídos de retinol sérico foram também encontrados em crianças de 18 a 119 meses de idade, atendidas, em São Paulo, no Hospital da Santa Casa de Misericórdia, com diarréia aguda; no trabalho, o grupo de estudo foi composto de 26 crianças e o grupo controle incluiu 52 crianças $^{75}$. As possíveis causas da diminuição do nível de retinol sérico observada nesse grupo foram atribuídas pelos autores a vários fatores, como mobilização diminuída da vitamina $A$ das reservas hepáticas, alteração ligada ao fenômeno da resposta de fase aguda decorrente da infecção, menor ingestão em virtude da anorexia, menor 
absorção intestinal, aumento da demanda e, ainda, aumento da excreção urinária, como ocorre em adultos com sepse e pneumonia.

\section{OUTROS ESTUDOS NACIONAIS}

O Estudo Nacional da Despesa Familiar (Endef, 1977-78)76 em meados da década de 70, foi realizado estudo de amplitude nacional, envolvendo áreas metropolitanas, áreas urbanas não metropolitanas e áreas rurais do Brasil. A pesquisa incluiu a avaliação da ingestão alimentar e algumas medidas antropométricas em cerca de 55 mil famílias habitantes das várias regiões do Brasil. O levantamento mostrou que, naquela época, o consumo médio de vitamina $A$, por indivíduo, encontrava-se aquém do recomendado em praticamente todas as regiões do país, com exceção das áreas metropolitanas do Rio de Janeiro e de Salvador.

No livro "Bóias-Frias": Uma Realidade Brasileira (1981) foram apresentados resultados da dosagem de vitamina A e caroteno séricos em trabalhadores volantes rurais ("bóias-frias") da área de Ribeirão Preto. Em uma das investigações, Angeleli et al. (1981) ${ }^{77}$ encontraram valores baixos de vitamina A plasmática em $2,1 \%$ de 48 indivíduos; em outro estudo, Desai et al. (1981) observaram níveis baixos de vitamina A plasmática em $25,0 \%$ de 124 indivíduos analisados. Foram, também, registrados níveis baixos e deficientes de caroteno sérico.

"A deficiência de vitamina A e estratégias para seu controle - um guia para as Secretarias Municipais de Saúde (1991)" foi publicado, em Fortaleza, Ceará, elaborado por membros das Universidades Federais do Ceará, Paraíba, Pernambuco e Bahia, que constituiu manual-guia para as Secretarias Municipais de Saúde, visando o controle da deficiência de vitamina A no Nordeste brasileiro. No manual, são abordados tópicos relacionados ao teor de vitamina $A$ dos alimentos, à deficiência da vitamina $A$ no
Nordeste, ao tratamento, à prevenção e à vigilância nutricional relacionadas à hipovitaminose $A^{79}$. No texto, estão incluídos dados sobre a prevalência da hipovitaminose $A$ em crianças carentes do Nordeste, participantes do Projeto Colaborativo de Vitamina A, desenvolvido no período de 1987 a 1990. Neste projeto, foram observados valores séricos de retinol abaixo de $20 \mu \mathrm{g} / \mathrm{dL}$ em $16,1 \%$ das crianças na região semiárida da Paraíba, 31,1\% no interior do Ceará, 34,1\% em Recife e 55,1\% na região semi-árida da Bahia.

O Estudo multicêntrico sobre consumo alimentar (INAN-MS/NEPA) aborda o resultado de um estudo multicêntrico desenvolvido através de pesquisa por amostragem probabilística de domicílios, coordenado pela Unicamp e UERJ e realizado nas cidades de Campinas, Curitiba, Goiânia, Ouro Preto e Rio de Janeiro, nos anos de 1996-1997. Incluiu o levantamento de dados socioeconômicos e demográficos, além do consumo de alimentos, considerando a família como unidade de análise, conforme metodologia proposta por Galeazzi et al. (1996) ${ }^{80}$. Foi, também, realizada a avaliação do consumo alimentar individual, utilizando questionário semi-quantitativo de freqüência de alimentos. A metodologia empregada permitiu conhecer o perfil de consumo da população envolvida e sua situação de risco nutricional referente a vários nutrientes, inclusive vitamina A. Na análise do consumo familiar, verificaram-se baixos valores de adequação de retinol para todas as faixas de renda na cidade de Ouro Preto, para todas as faixas de renda até dez salários mínimos per capita na cidade de Campinas e até a faixa de dois salários mínimos em Goiânia. Valores de adequação do consumo familiar de retinol ultrapassando $100 \%$ em todas as faixas de renda foram observados somente no Rio de Janeiro. Quanto ao consumo individual, considerando os participantes acima de 15 anos de idade, os valores de prevalência de inadequação não passaram de $6,1 \%$ da população de cada uma das cinco cidades estudadas, e esse problema afetou fundamentalmente o sexo 
feminino. Foi, também, assinalado que as famílias com renda até dois salários mínimos apresentavam risco nutricional de desenvolver deficiência de macro e micronutrientes, tendo sido apontado o retinol como um deles ${ }^{81}$.

\section{CONSIDERAÇÕ ES FINAIS}

A revisão dos trabalhos citados indica que o estado nutricional relacionado à vitamina A tem sido avaliado pela ingestão alimentar, pelo exame clínico, pela dosagem do retinol sérico e, com menor freqüência, pela determinação da concentração hepática de retinol. Assim, a hipovitaminose A tem sido diagnosticada a partir da ingestão deficiente de vitamina A, pelas lesões oculares, pelas concentrações séricas inferiores às aceitas universalmente como normais ou por concentrações hepáticas anormalmente diminuídas.

Desta maneira, a deficiência de vitamina A foi registrada em grupos populacionais de vários Estados brasileiros (Amazonas, Rio Grande do Norte, Paraíba, Pernambuco, Bahia, Minas Gerais, São Paulo e Santa Catarina), em várias capitais de Estados, em cidades grandes, como Ribeirão Preto e Campinas, e em cidades menores. Em algumas dessas regiões, a hipovitaminose $A$ foi reconhecida como um problema de saúde pública.

Número significativo dos estudos citados foi realizado com grupos populacionais de baixa renda. É mínimo o número de trabalhos relacionados com grupos populacionais de nível socioeconômico mais alto. Há, contudo, relatos de ingestão deficiente de vitamina $A^{41}$ e da existência de níveis séricos de retinol deficientes ou baixos ${ }^{51}$ mesmo em famílias de renda mais alta. Inexistência de associação entre a renda familiar per capita e prevalência de níveis deficientes de retinol sérico também foi constatada ${ }^{60,67}$. Contudo, Desai et al. (1984) ${ }^{47}$, em estudo com adolescentes pertencentes a famílias de alta e baixa renda, observaram adequação da ingestão de vitamina A de $131 \%$ e $50 \%$, respectivamente.
A propósito, no Third Report on the World Nutrition Situation $(1997)^{2}$, publicação do Subcomitê de Nutrição das Nações Unidas, é mencionado que a deficiência de vitamina A tem como causa habitual a baixa ingestão de vitamina A em relação aos requerimentos e que "a causa subjacente do consumo inadequado e das necessidades elevadas está relacionada à pobreza". O Fourth Report on the World Nutrition Situation ${ }^{4}$, publicado em janeiro de 2000 , ressalta ainda o fato de a deficiência de vitamina A estar ligada mais à natureza dos alimentos disponíveis e às práticas alimentares do que a "condições geológicas, químicas ou outras que afetam a população, como um todo, em diferentes áreas geológicas"; e complementa: "muitos estudos sugerem a existência de forte associação entre a deficiência de vitamina $A$ e condições socioeconômicas".

Quanto à associação entre a desnutrição energético-protéica e a hipovitaminose $A$, enquanto Gomes et al. (1970) ${ }^{14}$ e Varela et al. $(1972)^{15}$ observaram maior freqüência de valores mais baixos de retinol sérico em casos de desnutrição, Assis et al. (1997) ${ }^{59}$ e Ramalho et al. $(1998)^{62}$ não verificaram associação definida entre desnutrição e os níveis de retinol sérico.

No tocante à resposta de fase aguda, vários estudos avaliaram pacientes na presença de atividade inflamatória (varíola em crianças - Flores et al. (1984) ${ }^{46}$ e Campos et al. $(1987)^{74}$, infecções de vias aéreas superiores, pneumonia e diarréia - Velásquez-Meléndez et al. (1994) e Velásquez-Meléndez et al. (1996)75, observando-se diminuição do nível de retinol sérico. Neste sentido, em recente publicação, Stephensen \& Gildengorin (2000) ${ }^{82}$ verificaram que a presença da inflamação contribuiu para erros de classificação relacionados ao estado nutricional concernente à vitamina A. Assim, é importante considerar a atividade inflamatória para a interpretação correta dos níveis séricos de vitamina A. Isto se aplica em populações com prevalência elevada de infecções, mesmo aquelas subclínicas, as quais podem, por vários mecanismos, deter- 
minar queda da concentração sérica de retinol, sem que isso represente um comprometimento do estado nutricional relacionado com a vitamina $A^{83}$. De fato, segundo estes autores, a diminuição da concentração de retinol sérico, na vigência de doenças, pode não refletir com exatidão o estado nutricional relacionado com a vitamina em populações com alta prevalência de infecções. Vários fatores podem participar da diminuição na concentração sérica de retinol durante as infecções, como necessidade aumentada da vitamina, sua distribuição entre tecidos e aumento de sua utilização ${ }^{83}$.

A partir dos resultados dos estudos enfocando as manifestações, é possível classificá-los em dois grupos: aqueles que detectaram lesões oculares incluindo a cegueira noturna, as manchas de Bitot e a xerose conjuntival, relatadas em maior número de trabalhos ${ }^{12,15,22,24-27,34,38,43-45,56}$, e aqueles que abordam a xerose corneana e cicatrizes da córnea. Lesões deste tipo foram relatadas em investigações de Roncada et al. $(1975)^{22}$, no Estado de São Paulo, Santos et al. (1983a, 1983b) ${ }^{43,44}$ e Araujo et al. (1984)45, na Paraíba. Na década de 70, Simmons \& Mello $(1975)^{25}$ e Simmons $(1976)^{26}$ referiram a alta freqüência de cegueira por deficiência de vitamina A no Nordeste brasileiro. Esse estudo, entretanto, ressente-se do aspecto metodológico e suas conclusões foram contestadas por investigação posterior, através de inquérito realizado com oftalmologistas brasileiros, o qual mostrou baixa prevalência das lesões consideradas significativas da xeroftalmia ${ }^{24}$. Desta maneira, as evidências parecem indicar que a xeroftalmia não representava problema de saúde pública no Brasil. McAuliffe et al. (1991) ${ }^{79}$ registraram no período de 1990 a 1991, a partir da vigilância em 492 leitos hospitalares, em hospitais pediátricos de João Pessoa, sete casos de xeroftalmia. Em conclusão, no Brasil, a questão parece não se centrar em torno de lesões oculares significativas de deficiência da vitamina; há, na realidade, abundância de dados indicando a existência de sinais oculares leves atribuíveis à deficiência de vitamina $\mathrm{A}$.
Em síntese, este artigo traz evidências de que as manifestações clínicas de deficiência de vitamina A correspondem fundamentalmente a lesões oculares, observadas com maior freqüência na Região Nordeste; adicionalmente, notam-se as altas proporções de crianças com níveis bioquímicos inadequados na maioria das regiões estudadas. Nos últimos 20 anos, ganhou importância a noção da deficiência subclínica da vitamina $A$, pois esta se associa a elevados riscos de mortalidade e morbidade, principalmente em crianças $^{84}$. De fato, conforme indicam relatos concludentes, o aumento da morbi-mortalidade ocorre mesmo antes da instalação de cegueira noturna e xeroftalmia, manifestações clínicas da deficiência de vitamina $A^{8}$.

Pode-se concluir que a deficiência de vitamina A constitui um problema de saúde pública em várias regiões do Brasilis8-60,67. Embora a maioria dos estudos sobre a hipovitaminose $A$ enfoque crianças de idade pré-escolar, alvo preferencial da deficiência, é possível que essa deficiência constitua um problema importante de saúde para crianças em idade escolar27,33,34,43,49,50,53,56,75. Por outro lado, de acordo com os resultados de alguns estudos, a hipovitaminose A não tem importância epidemiológica para os indivíduos adultos 21,22,24,34,35.

\section{REFERÊ NCIAS}

1. Sommer A, West KP. Vitamin deficiency: health, survival, and vision. New York: Oxford University Press; 1996.

2. Third Report on the World Nutrition Situation. Administrative Committee on Coordination/Sub-Committee on Nutrition. Geneva: Publications WHO; 1997. 111p.

3. International Vitamin A Consultative Group. Maternal night blindness: extent and associated risk factors. An IVACG Statement. Washington DC; 1997.

4. Fourth Report on the World Nutrition Situation. Administrative Committee on Coordination/Sub- 
-Committee on Nutrition. Geneva: Publications WHO; 2000. $121 \mathrm{p}$.

5. World Health Organization. Indicators for assessing vitamin A deficiency and their application in monitoring and evaluating intervention programmes. Geneva; 1996. (Micronutrient Series, 10).

6. Tomkins AM, Behrens R, Roy S. The role of zinc and vitamin A deficiency in diarrhoeal syndromes in developing countries. Proc Nutr Soc 1993; 52:131-42.

7. Velasquez-Melendez G, Okani ET, Riertsman B, Roncada MJ. Níveis plasmáticos de vitamina A, carotenóides e proteína ligadora de retinol em crianças com infecções respiratórias agudas e doenças diarréicas. Rev Saúde Pública 1994; 28:357-64.

8. Bloem MW, De Pee S, Danton-Hill ID. New issues in developing effective approaches for the prevention and control of vitamin A deficiency. Food Nutr Bull 1998; 19:137-48.

9. Dutra de Oliveira JE, Marchini JS. Levantamento bibliográfico de estudos bioquímicos-nutricionais sobre micronutrientes realizados no Brasil. Cad Nutr 1994; 8:31-67.

10. Dutra de Oliveira JE, Cunha SFCC, Marchini JS. A desnutrição dos pobres e dos ricos: dados sobre a alimentação no Brasil. São Paulo: Sarvier; 1996 $123 p$.

11. Arroyave G, Chichester CO, Flores H, Glover JG, Mejía LA, Olson JA, etal. Biochemical methodology for the assessment of vitamin A status. Washington DC: IVACG; 1982. 88p.

12. Pereira NDV, Abreu LV, Freusberg O. Observações clínicas em 64 crianças portadoras de hipovitaminose A. Arq Catarin Med 1966; 1:16-26.

13. Batista Filho M, Gomes SMF. Níveis séricos de vitamina A e caroteno em diferentes grupos etários. Hospital 1969; 76:73-8.

14. Gomes FS, Batista M, Varela RM, Bazante MO Salzano AC. Plasma retinol levels of pre school children in the sugar-cane area northeast Brazil. Arch Latinoam Nutr 1970; 20:445-51.
15. Varela RM, Teixeira SG, Batista Filho M. Hipovitaminosis $A$ in the sugar cane zone of Southern Pernambuco state, Northeast Brazil. Am J Clin Nutr 1972; 25:800-4.

16. Roncada MJ. Hipovitaminose "A". Níveis séricos de vitamina " $A$ " e caroteno em populações litorâneas do Estado de São Paulo, Brasil. Rev Saúde Pública 1972; 6:3-18.

17. Batista Filho M, Teixeira SFG, Linhares EDR. Retinol sérico de gestantes atendidas em serviços de Saúde Pública. Rev Bras Pesqui Med Biol 1973; 6: $229-34$

18. Bon AMX, Miguel M. O consumo de vitamina A em Ribeira, São Paulo (Brasil). Rev Saúde Pública $1974 ; 8: 87-92$.

19. Mazzilli RN. Algumas considerações sobre o consumo de alimentos em Icapara e Pontal de Ribeira, São Paulo, Brasil. Rev Saúde Pública 1975; 9:49-55.

20. Martins IS, Cavalcanti MLF, Mazzilli RN. Relação entre consumo alimentar e renda familiar na cidade de Iguape, São Paulo (Brasil). Rev Saúde Pública 1977; 11:27-38.

21. Roncada MJ. Inquérito entre migrantes atendidos pela Central de Triagem e Encaminhamento, na capital do Estado de São Paulo, Brasil. II Aspectos bioquímicos da hipovitaminose. Rev Saúde Pública 1975; 9:313-29.

22. Roncada MJ, Wilson $D$, Netto $A L$, Netto $O B$, Kalil AC, Nunes MF, et al. Hipovitaminose A em filhos de migrantes nacionais em trânsito pela capital do Estado de São Paulo, Brasil. Estudo clínico-bioquímico. Rev Saúde Pública 1978; 12:345-50.

23. Roncada MJ. Inquérito entre migrantes atendidos pela Central de Triagem e Encaminhamento, na capital do Estado de São Paulo, Brasil. III. Aspectos alimentares. Rev Saúde Pública 1975; 9:507-14.

24. Roncada MJ, Wilson D, Netto AL, Netto RB, Kalil AC. Investigação sobre a prevalência de xeroftalmia através de inquérito realizado junto a oftalmologistas brasileiros. Rev Saúde Pública 1978; 12:151-56. 
25. Simmons WK, Mello A. Blindness in the nine states of northeast, Brazil. Am J Clin Nutr 1975; 28:202.

26. Simmons WK. Xerophtalmia and blindness in Northeast Brazil. Am J Clin Nutr 1976; 29:116-22.

27. Giugliano R, Albuquerque HCR, Shrimpton R. Estudo antropométrico, clínico e de padrões alimentares em um grupo de escolares de Manaus, 1976. Acta Amazon 1978; 8:75-82.

28. Shrimpton R, Giugliano R. Consumo de alimentos e alguns nutrientes em Manaus, Amazonas. Acta Amazon 1979; 9:117-41.

29. Araújo RL, Souza MSL, Mata-Machado AJ, Mata-Machado LT, Mello ML, Costa Cruz TA, et al. Response of retinol serum levels to the intake of vitamin A fortified sugar by pre-school children. Nutr Rep Int 1978; 17:307-14.

30. Carvalho PB, Dutra de Oliveira JE. Disponibilidade de vitamina " $A$ " na alimentação de um grupo de pré-escolares na cidade de Ribeirão Preto. ABIA/SAPRO 1979; 44:18-28.

31. Ward JQ, Amoni D. Diets of urban migrants in northeast Brazil: an economic analysis. Nutr Rep Int 1980; 21:11-23.

32. Desai ID, Garcia Tavares ML, Dutra de Oliveira BS, Douglas A, Duarte FAM, Dutra de Oliveira JE. Food habits in southern Brazil. Am J Clin Nutr 1980; 33:702-14.

33. Wilson D, Roncada MJ, Mazzilli RN, Cavalcanti MLF, Pattoli DBG. Nutritional status of children, inmates of a small institution for homeless children in the capital of the state of São Paulo, Brazil. Rev Saúde Pública 1980; 14:300-9.

34. Roncada MJ, Wilson D, Mazzilli RN, Gandra YR. Hipovitaminose A em comunidades do Estado de São Paulo, Brasil. Rev Saúde Pública 1981; 15 : 338- 49.

35. Roncada MJ, Marucci MFN, Lamonica IMR. Níveis sangüíneos de vitamina A e caroteno em indivíduos de meia-idade e idosos, em onze localidades do Estado de São Paulo, Brasil. Rev Saúde Pública 1985; 19:336-43.

36. Borges EL, Araujo RL, Oliveira JR. Efeito de ingestão de açúcar fortificado com vitamina A (50Ul/g) no combate à hipovitaminose A. Rev Assoc Med Bras $1981 ; 27: 234-36$.

37. Horner MR, Dorea JG, Pereira MG, Bezerra VL, Salomon JB. Inquérito dietético com base no consumo familiar: o caso de Ilhéus, Bahia, Brazil, em 1979. Arch Latinoam Nutr 1981; 31:727-39.

38. Giugliano R, Giugliano LG, Shrimpton R. Estudos nutricionais das populações rurais da Amazônia. I. Várzea do Rio Solimões. Acta Amazon 1981; 11:773-88.

39. Araújo DS, Shrimpton R. Padrão alimentar e consumo de zinco, vitamina A e ferro, em pré-escolares num bairro pobre de Manaus, 1979. Acta Amazon 1982; 12:591-97.

40. World Health Organization. Requirements of vitamin A, thiamin, riboflavin and niacin. Report of a Joint FAO/WHO expert group. Geneva; 1967. $86 p$.

41. Shrimpton R. Food consumption and dietary adequacy according to income in 1.200 families, Manaus, Amazons, Brazil, 1973-1974. Arch Latinoam Nutr 1984; 34:615-29.

42. Marinho HA, França TS, Rebelo YS, Shrimpton NR. Níveis séricos de vitamina " $A$ " em operários de Manaus, Amazonas. Acta Amazon 1981; 11: 347-53.

43. Santos LMP, Dricot JM, Asciutti LS, Benigna MJCN, Dricot D’Ans C. Estudo epidemiológico da xeroftalmia no Estado da Paraíba. Rev Bras Med 1983a; 40:419-25.

44. Santos LMP, Dricot JM, Asciutti LS, Dricot-D'ans C. Xerophtalmia in the state of Paraíba, northeast of Brazil: Clinical findings. Am J Clin Nutr 1983b; 38:139-44.

45. Araújo RL, Diniz AS, Santos LMP. Diagnóstico e evolução de casos de ceratomalácia e xeroftalmia. J Pediat 1984; 57:419-24.

46. Flores H, Campos F, Araujo CRC, Underwood BA. Assessment of marginal vitamin A deficiency in Brazilian children using the relative dose response procedure. Am J Clin Nutr 1984; 40:1281-89.

47. Desai ID, Wadell C, Dutra S, Dutra de Oliveira S, Duarte $\mathrm{E}$, Robazzi ML, et al. Marginal malnutrition 
and reduced physical work capacity of migrant adolescent boys in Southern Brazil. Am J Clin Nutr 1984; 40:135-45.

48. Roncada MJ, Wilson D, Okani ET, Amino S. Prevalência de hipovitaminose $A$ em pré-escolares de municípios de área metropolitana de São Paulo, Brasil. Rev Saúde Pública 1984; 18:218-224.

49. Araujo RL, Araujo BDG, Siero RO, Machado RDP, Leite BV. Diagnóstico da situação da hipovitaminose $A$ e da anemia nutricional na população do Vale do Jequitinhonha, Minas Gerais, Brasil. Arch Latinoam Nutr 1986; 36:642-53.

50. Araújo RL, Araújo MBDG, Machado RDP, Braga $A A$, Leite BV, Oliveira JR. Evaluation of a program to overcome vitamin $A$ and iron deficiencies in areas of poverty in Minas Gerais, Brazil. Arch Latinoam Nutr 1987; 37:9-22.

51. Romani SA, Amigo H. Perfil alimentar e posse de terra na área rural do Estado de Pernambuco, Nordeste do Brasil. Rev Saúde Pública 1986; 20:369-76.

52. Lira PIC, Cartagena HA, Romani SA, Torres MAA, Batista Filho M. Estudo nutricional de crianças menores de seis anos, segundo posse de terra, em áreas rurais do Estado de Pernambuco, Nordeste do Brasil. Arch Latinoam Nutr 1985; 35:247-57.

53. Rivera MAA, Rivera FA. Perfil nutricional da dieta de escolares e pré-escolares pertencentes à população de baixa renda. Ciênc Cult Saúde 1986; 8:12-4.

54. Favaro RMD, Souza NV, Batistal SM, Ferriani MEC, Desai ID, Dutra de Oliveira JE. Vitamin A status of young children in southern Brazil. Am J Clin Nutr 1986; 43:852-8.

55. Favaro RMD, Souza NV, Vannucchi H, Desai ID, Dutra de Oliveira JE. Evaluation of rose bengal staining test and rapid dark-adaptation test for the field assessment of vitamin A status of preschool children in southern Brazil. Am J Clin Nutr 1986; 43:940-5

56. Mariath JGR, Lima MCC, Santos LMP. Vitamin A activity of Buriti (Mauritia Vinifera Mart) and its effectiveness in the treatment and prevention of xerophtalmia. Am J Clin Nutr 1989; 49:849-53.
57. Moura EC, Rossi AVT, Sanches AL, Vendramini CM, Françoso TA. Perfil nutricional de gestantes atendidas no Centro de Saúde Escola Jardim Novo Campos Elíseos da PUCCAMP. Rev Nutr PUCCAMP 1990; 3:113-36.

58. Prado MS, Assis AMO, Martins MC, Nazaré MPA, Rezende IFB, Conceição MEP. Hipovitaminose A em crianças de áreas rurais do semi-árido baiano. Rev Saúde Pública 1995; 29:295-300.

59. Assis AMO, Prado MS, Freitas MCS, Cruz MM. Deficiência de vitamina $A$ e desnutrição energético-protéica em crianças de localidades do semi-árido baiano. Rev Nutr PUCCAMP 1997; 10: 70-8.

60. Santos LMP, Assis AMO, Martins MC, Araujo MPN, Morris SS, Barreto ML. Situação nutricional e alimentar de pré-escolares no semi-árido da Bahia (Brasil): II - Hipovitaminose A. Rev Saúde Pública 1996; 30:67-74.

61. Velásquez-Meléndez G, Martins IS, Cervato AM, Fornés NS, Marucci MFN. Consumo alimentar de vitaminas e minerais em adultos residentes em área metropolitana de São Paulo, Brasil. Rev Saúde Pública 1997; 31:157-62.

62. Ramalho RA, dos Anjos LA, Flores H. Hipovitaminose $A$ em recém-nascidos em duas maternidades públicas no Rio de Janeiro, Brasil. Cad Saúde Pública 1998; 14:821-27.

63. Vitolo MR, Accioly E, Ramalho RA, Soares AC, Cardoso CB, Carvalho EB. Níveis de vitamina A no leite maduro de nutrizes adolescentes e adultas de diferentes estratos socioeconômicos. Rev Ciênc Med 1999; 8:3-10.

64. Rondó PHC, Abbott R, Rodrigues LC, Tomkins AM. Vitamin A, folate, and iron concentrations in cord and maternal blood of intra-uterine growth retarded and appropriate birth weight babies. Eur J Clin Nutr 1995; 49:319-99.

65. Rondó PHC, Abbott R, Tomkins AM. Vitamin A and neonatal antropometry. J Trop Pediatr 2001; 47:307-10.

66. Rondó PHC, Villar BS, Tomkins AM. Vitamin A status of pregnant women assessed by a biochemical indicator and a simplified food 
frequency questionnaire. Arch Latinoamer Nutr 1999; 49:322-25.

67. Ferraz IS, Daneluzzi JCS, Vannucchi H. Vitamin A deficiency in children aged 6 to 24 months in São Paulo state, Brazil. Nutr Res 2000; 20:757-68.

68. Olson JA. Liver vitamin A reserves of neonates, preschool children and adults dying of various causes in Salvador, Brazil. Arch Latinoam Nutr 1979; 29:521-45.

69. Flores $H$, Araujo CRC. Liver levels of retinol in unselected necropsy specimens: a prevalence survey of vitamin A deficiency in Recife, Brazil. Am J Clin Nutr 1984; 40:146-52.

70. Dorea JG, Souza JA, Galvão MO, lunes MAF. Concentration of vitamin $A$ in the liver of foetuses and infants dying of various causes in Brasilia, Brazil. Int J Vitam Nutr Res 1984; 54:119-23.

71. Araújo RL, Borges EL, Silva J, D’Arck B, Vieira EC. Failure of massive oral doses of vitamin $A$ to prevent hypovitaminosis. Nutr Rep Int 1978; 18:503-13.

72. Souza MSL, Carvalho OS, Souza OWC, Massara $\mathrm{CL}$, Araujo RL, Paulino NMS, et al. Inter-relação entre parasitosis e hipovitaminose A. Infecção por Shistosoma mansoni e o nível sérico de retinol de população de zona endêmica de Minas Gerais (Brasil). Rev Inst Med Trop 1988; 30:281-7.

73. Marinho HA, Shrimpton R, Giugliano R, Burini RC. Influence of enteral parasites on the blood vitamin A levels in preschool children orally supplemented with retinol and/or zinc. Eur J Clin Nutr 1991; 45:539-44

74. Campos FACS, Flores $\mathrm{H}$, Underwood BA. Effect of an infection on vitamin $A$ status of children as measured by the relative dose response (RDR). Am J Clin Nutr 1987; 46:91-4.

75. Velasquez-Melendez G, Roncada MJ, Toporovski J, Okani ET, Wilson D. Relationship between acute diarrhoea and low plasma levels of vitamin $A$ and retinol binding protein. Rev Inst Med Trop São Paulo 1996; 38:365-9.

76. Fundação Instituto Brasileiro de Geografia e Estatística. Estudo Nacional da Despesa Familiar: Consumo alimentar. Despesas das famílias. Dados preliminares, tabelas selecionadas. Rio de Janeiro; 1977-78. p.124.

77. Angeleli WA, Moura Duarte FA, Dutra de Oliveira JE. Estado nutricional, alimentação e capacidade física de trabalhadores volantes rurais ou "bóias-frias". In: Dutra de Oliveira JE, editor. Bóias-Frias: uma realidade brasileira. São Paulo: Publicação ACIESP; 1981. p.7-85.

78. Desai ID, Tavares MLG, Dutra de Oliveira BS, Douglas A, Duarte FAM, Dutra de Oliveira JE. Hábitos alimentares e estado nutricional das famílias dos "bóias-frias" ou trabalhadores volantes da área de Ribeirão Preto. In: Dutra de Oliveira JE, editor. Bóias-Frias: uma realidade brasileira. São Paulo: Publicação ACIESP; 1981. p.86-116.

79. Mcauliffe J, Santos LMP, Diniz AS, Batista Filho M, Barbosa RCC. A deficiência de vitamina A e estratégias para seu controle: um guia para as secretarias municipais de saúde. Fortaleza; 1991. p.29. Projeto Hope.

80. Galeazzi MAM. Inquérito de consumo familiar. Metodologia para identificação de famílias de risco alimentar. Cad Debate 1996; 4:32-46.

81. Galeazzi MAM, Domene SMA, Sichieri R. Estudo multicêntrico sobre consumo alimentar. Cad Debate 1997; (Volume Especial):11-60.

82. Stephensen CB, Gildengorin G. Serum retinol, the acute phase response, and the apparent misclassification of vitamin A status in the third National Health and Nutrition Examination Survey. Am J Clin Nutr 2000; 72:1170-8.

83. Filteau SM, Morris SS, Abbott RA, Tomkins AM, Kirkwood BR, Arthur P, et al. Influence of morbidity on serum retinol of children in a community-based study in northern Ghana. Am J Clin Nutr 1993; 58:192-97.

84. Velásquez-Meléndez G, Roncada MJ. Deficiência de vitamina $A$ e sua relação com a morbi-mortalidade infantil: aspectos epidemiológicos. Cad Nutr 1994; 8:10-18.

Recebido para publicação em 11 de maio de 1999 e aceito em 13 de dezembro de 2002. 